\title{
The sun illusion: Individual differences in remembered size and distance judgments
}

WILLIAM L. KING AND MICHAEL C. HAYES

DALHOUSIE UNIVERSITY

College students judged, from memory, the relative size of the sun at the horizon and at the zenith, the relative distance to the sun at each position, and the relative distance to the sky at each position. Two major types of $S$ s were identified. The more numerous type remembered the sun at the horizon as larger and closer than the zenith sun; the other remembered the sun at the horizon as larger and more distant than the zenith sun. For the more numerous type, the size and distance judgments were negatively correlated; for the other type they were positively correlated.

Recent investigations of the moon illusion have resurrected the Ptolemaic hypothesis which states that the horizon moon appears larger than the zenith moon because the horizon appears further away than the zenith (King \& Gruber, 1962; Kaufman \& Rock, 1962; Rock \& Kaufman, 1962; Gruber, King, \& Link, 1963; McNulty \& St. Claire-Smith, 1964). The horizon moon further away, yet subtending the same visual angle as the zenith moon, appears larger. The "catch" to this line of reasoning is that the horizon moon appears closer than the zenith moon to most observers. Rock \& Kaufman (1962) suggest that, while distance correlates are important determinants of the size illusion, the phenomenal size itself is the primary determinant of the distance judgment. The paradox is resolved thusly: the stronger the cues for distance to the horizon, the larger, and therefore the closer, the horizon moon will appear.

The present study is a step toward investigating individual differences in the perception of size and distance. The fact is, that while Rock \& Kaufman's (1962) interpretation has much to recommend it, it may describe only the most numerous of different types of persons. The fact that some individuals report no moon illusion and that judgments of size and distance can show tremendous variation suggests that investigating individual differences may be as informative as investigating the typical mode of responding.

Specifically, it was hypothesized that the typical $S$ would report the horizon moon to be larger and closer, although the distance to the horizon would be reported to be greater than the distance to the zenith. However, it was also hypothesized that a smaller number of Ss would report the horizon moon as larger and further away as well as reporting the distance to the horizon to be greater than the distance to the zenith. In other words, some Ss would "use" apparent size as the cue for apparent distance while others would "use" more directly related distance cues. The sun illusion was used rather than the moon illusion since the two are very similar and since preliminary work suggested that Ss found judgments of the sun easier to make than judgments of the moon.

\section{Method}

The Ss were 347 students in an introductory psychology class who were naive with regard to the problem. They were required to fill out a free response questionnaire asking for judgments of the relative size and distance of the sun at the zenith and at the horizon. In addition, Ss were asked to draw the shape of the sky from horizon to horizon. The purpose of this last question was to find out whether the horizon was remembered as being further away than the zenith. To help obviate the effects of order, all six possible orders of the three judgments were represented. A description of the judgment and examples of some possible responses were provided. The examples always included "opposite" types of drawings so as not to bias $S^{\prime}$ 's representation. For the distance judgment $S$ drew two lines, for which the ratio of the longer to the shorter was equal to the ratio of distances for the sun at the horizon and at the zenith. For the size judgments $S$ drew two circles, for which the ratio of the greater diameter to the lesser was equal to the ratio of diameters of the sun at the horizon and at the zenith. The shape of the sky was drawn as a line extending from horizon to horizon above a small stick figure (the positions of the horizons were marked on the paper). Results

The data obtained from each of the three drawings were expressed as horizon/zenith $(\mathrm{H} / \mathrm{Z})$ ratios. The diameter for the horizon sun was divided by the diameter for the zenith sun to produce the $\mathrm{H} / \mathrm{Z}$ ratio for the size judgments. The larger the illusion the more this ratio will exceed unity. Similarly, the $\mathrm{H} / \mathrm{Z}$ ratio for the sky judgments (distance to the horizon and to the zenith) was computed. The more this ratio exceeds unity the further away is the horizon as compared to the zenith. Finally, judgments of the distance to the sun yield a third $\mathrm{H} / \mathrm{Z}$ ratio. The extent by which this ratio is smaller than unity reflects the extent by which the horizon sun is judged as closer than the zenith sun.

The mean, median, mode, and standard deviation for each judgment are shown in Table 1 under the column headed "Total." The magnitude of the mean $\mathrm{H} / \mathrm{Z}$ ratio for the size judgments accords well with those obtained in previous studies of the moon illusion. Moreover, the median values describe the typical observer remembering the horizon sun as approximately half again as large 
Table 1. H/Z Ratios

\begin{tabular}{|c|c|c|c|c|c|c|c|c|c|}
\hline & \multicolumn{3}{|c|}{$\begin{array}{l}\text { Size } \\
\text { Judgments }\end{array}$} & \multicolumn{3}{|c|}{$\begin{array}{l}\text { Distance } \\
\text { Judgments }\end{array}$} & \multicolumn{3}{|c|}{$\begin{array}{l}\text { Sky } \\
\text { Judgments }\end{array}$} \\
\hline & 1 & 117 & Total* & 1 & 11 & Total* & 1 & 111 & Total* \\
\hline Mean & 1.67 & 1.72 & 1.53 & 1.58 & .57 & .95 & 1.96 & 2.03 & 2.07 \\
\hline SD & .41 & .57 & .62 & .50 & .17 & .56 & 1.73 & 1.42 & 1.43 \\
\hline Mdn & 1.58 & 1.64 & 1.50 & 1.52 & .58 & .72 & 1.39 & 1.30 & 1.35 \\
\hline Mode & 1.50 & 1.50 & 1.00 & 1.48 & .59 & 1.00 & 1.09 & $* *$ & $* *$ \\
\hline$N$ & 70 & 184 & 347 & 70 & 184 & 346 & 70 & 184 & 345 \\
\hline
\end{tabular}

* Total includes scores from all subjects including those not classifiable as Type I or Type II because they did not complete one or more of the judgments (9\%), had no illusion (7\%) or had a negative illusion (12\%). Since all ss did not complete all judgments, the total $N^{\prime} s$ are not exactly equal.

** These distributions were multimodal.

in diameter as the zenith sun and at two-thirds the distance. This same observer remembers the sky as a flattened dome whose zenith is approximately twothirds the distance to the horizon. However, this pattern reflects only the more numerous of two subtypes. Ss were divided into two types, both consisting of Ss who remembered the horizon sun as larger than the zenith sun. For Type I, however, the horizon sun was more distant than the zenith sun, and for Type II the horizon sun was closer. Type I therefore represents those Ss whose judgments are in accord with a one-stage process in which the same factors responsible for increased size also increase the distance. Type II represents those

Table 2. Correlations Between $\mathbf{H} / \mathbf{Z}$ Ratios for Size, Distance and Sky Judgments

\begin{tabular}{lccc} 
Variables & $\begin{array}{c}\text { Type I } \\
(\mathrm{N}=70)\end{array}$ & $\begin{array}{c}\text { Type II } \\
(\mathrm{N}=184)\end{array}$ & $\begin{array}{c}\text { Type I + Type II } \\
(\mathrm{N}=254)\end{array}$ \\
\hline Size and distance $.298^{* *}$ & $-.167^{*}$ & .001 \\
Size and Sky & .052 & .040 & .044 \\
Distance and Sky-. 166 & .000 & -.051 \\
\hline
\end{tabular}

$* p<.05$

$* * p<.01$
Ss whose judgments are in accord with a two-stage process in which the distance correlates produce the increased size, which in turn produces a decreased distance.

For Type I and Type II Ss the relationship between the distance judgments and the size judgments should be in opposite directions. One would expect a positive correlation between the size and distance judgments for the Type I Ss, and a negative relationship for the Type II Ss. Table 2 shows the correlations between the size ratios, the distance ratios, and the sky ratios for Type I, Type II and the combined group. The prediction is confirmed as shown by the fact that the correlations between distance and size are roughly equal in magnitude for both types but are opposite in sign. None of the correlations with the sky ratios are significant, which is not surprising in light of the enormous variability associated with this variable. Moreover, people seemed to find sky judgments more difficult than size or distance judgments.

The results are sufficiently provocative to warrant further study. However, the obtained relationships, while significant, account for little variance. Whether the level of relationship is limited by the nature of the processes involved or simply drowned out by the error of measurement can only be determined by further investigation. Finally, Ss may not see what they remembered seeing.

\section{References}

Gruber, H. E., King, w. L., \& Link, S. Moon illusion: An event in imaginary space. Science, 1963, 139, 750-752.

Kaufman, L., \& Rock, I. The moon illusion, I. Science, 1962, 136, 953-961.

King, W. L., \& Gruber, H. E. Moon illusion and Emmert's Law, Science, 1962, 135, 1125-1126.

McNulty, J. A., \& St. Claire-Smith, R. Terrain effects upon perceived distance. Canad. J. Psychol., 1964, 18, 175-182.

Rock, I., \& Kaufman, L. The moon illusion, II. Science, 1962, $136,1023-1031$. 